\title{
Article
}

\section{Estimation of Radionuclide Intakes by Singular Value Decomposition}

\author{
Shinji HATO ${ }^{1,2, *}$ and Sakae KINASE ${ }^{1,3}$ \\ ${ }^{1}$ Institute of Applied Beam Science, Graduate School of Science and Engineering, Ibaraki University, 2-1-1 Bunkyo, Mito-shi, \\ Ibaraki 310-8512, Japan \\ ${ }^{2}$ Visible Information Center, Inc., 440 Muramatsu, Tokai-mura, Naka-gun, Ibaraki 319-1112, Japan \\ ${ }^{3}$ Fukushima Environmental Safety Center, Japan Atomic Energy Agency, 2-4 Shirane, Shirakata, Tokai-mura, Naka-gun, \\ Ibaraki 319-1195, Japan
}

\begin{abstract}
It is important to accurately estimate the intake quantity for reliable internal exposure assessments. The intake quantity has been estimated by using the least-squares method. However, to use the least-squares method, the number of radioactivity measurements must be more than the number of intakes. To remedy this restriction, this study suggests an estimation method using singular value decomposition that is available regardless of the relation between the numbers of measurements and intakes. Moreover, this study introduces a procedure to calculate the intake quantity from the measurements with uncertainty.
\end{abstract}

KEYWORDS: internal exposure, intake estimation, radioactivity measurement, singular value decomposition

\section{Introduction}

Internal exposure occurs by the intake of radioactive materials into the human body and continues until the radioactive materials are excreted or attenuated by decay. Internal exposure can have a severe impact on the human body as the internal organs are directly exposed to the radiation. Therefore, it is necessary to consider the effects of not only strongly penetrating $\gamma$-rays, but also weakly penetrating radiation such as $\alpha$-rays emitted from the progeny nuclides produced during radioactive decay inside the human body, to evaluate their effects.

The effects of internal exposure on the human body are evaluated by the committed equivalent dose in the internal organs or the committed effective dose in the entire body accumulated over 50 years for adults and until 70 years old for children ${ }^{1)}$. These internal exposure doses are generally evaluated by multiplying the dose coefficient $[\mathrm{Sv} / \mathrm{Bq}]$, calculated using an internal exposure evaluation model devised by the International Commission on Radiological Protection (ICRP), by the intake quantity of radioactive material in the body [Bq]. Accordingly, to evaluate the internal exposure dose with a high degree of accuracy, not only the physicochemical characteristics (such as the particle size of the inhaled radioactive materials, their chemical

* Corresponding author, E-mail: hato@vic.co.jp

DOI : 10.15669/fukushimainsights.Vol.4.501

(C) 2021 Atomic Energy Society of Japan. All rights reserved.

Originally published in Transactions of the Atomic Energy Society of Japan (ISSN 1347-2879), Vol. 15, No. 3, p.146-150

(2016) in Japanese. (Japanese version accepted: March 14, 2016) 
form and the precise dose coefficient based on the exposure situation (the activity status of the examinee)) but also an evaluation of the precise intake quantity, become necessary.

The unknown intake quantity of radioactive materials due to an accident is estimated using the predicted radioactivity per unit intake and the measured radioactivity in the body or excretions after an accident. According to the ICRP publication 78 that provides a method for evaluating the internal exposure of workers ${ }^{2)}$, it is suggested that a single intake can be estimated by dividing the radioactivity measurement by the radioactivity prediction for a unit intake. Furthermore, in the IDEAS guidelines that describe the evaluation procedures for internal exposures ${ }^{3)}$, it is suggested that a single intake can be estimated from multiple measurements in which the uncertainly of measurement is considered. Generally, the intake estimation is obtained by fitting the radioactivity prediction structured with a linear combination of radioactivity prediction for the unit intake and the unknown intake quantity to the radioactivity measurement in the body or excretions. Accordingly, the estimated intake quantity is formulated as a least squares problem. The least squares method for solving this problem is only applicable when the intake frequency is lower than the number of radioactivity measurements, and there is a limit on the type of applications.

In this paper, a methodology using singular-value decomposition that allows estimation of the intake quantity using multiple intakes and multiple measurements with uncertainty is proposed. The results of the application of this estimation method to case examples in previous studies are reported.

\section{Internal Radioactivity Prediction Formula}

\section{Convolution Integration}

The internal exposure dose due to a single acute intake in the ICRP is evaluated by the multiplication of the intake quantity and dose coefficient. This dose coefficient indicates the internal exposure dose using the biokinetic model and the ICRP dose evaluation model, for the case where an acute intake of $1 \mathrm{~Bq}$ of radioactive nuclides occurred at an arbitrary age. The biokinetic model evaluates the metabolism of radioactive nuclides and predicts the radioactivity from an intake of $1 \mathrm{~Bq}$ (in the body or in excretions), i.e., the radioactivity prediction per unit intake. The internal radioactivity by chronic intake of radioactive nuclides is calculated by convolution integration on the intake rate and the radioactivity prediction per unit intake:

$$
a(T)=\int_{0}^{T} \dot{I}(t) \times q(T-t) d t
$$

where, $a(T)$ is the radioactivity prediction $[\mathrm{Bq}]$ at the age in days $T[d], \dot{I}(t)$ is the intake rate of radioactive nuclides $[\mathrm{Bq} / \mathrm{d}]$, and $q(T-t)$ is the radioactivity prediction $[\mathrm{Bq} / \mathrm{Bq}]$ per unit intake at the age of $T(T>t)$ in days when an intake occurred at the age of $t$ in days.

\section{Discretization of Integration}

By conducting convolution integration on the radioactivity prediction per unit acute intake and the intake rate of the radioactive nuclides, the radioactivity prediction of the chronic intake can be calculated as Eq. (1). Using the average intake quantity per day $\left(I\left(t_{j}\right)=\dot{I}\left(t_{j}\right) \Delta t_{j}, \Delta t_{j}=1\right)$, the integration of Eq. (1) is discretized: 


$$
a\left(T_{i}\right)=\sum_{j} q\left(T_{i}-t_{j}\right) I\left(t_{j}\right)
$$

Using Eq. (2), the prediction of radioactivity due to the chronic intake (multiple number of intakes) is evaluated based on the intake quantity expressed per the unit of one day and the prediction of radioactivity due to a single acute intake is acquired when $j=1$. Accordingly, it is considered that the intake quantity is acquired by solving Eq. (2), but it is not possible to solve as the number of unknown quantities (number of intakes) and the number of equations (number of measurements) do not necessarily match. Next, a methodology is considered for estimation of intake quantity based on the radioactivity measurements.

\section{Intake Quantity Estimation Method}

\section{Deriving the Intake Quantity Estimation Formula}

The intake quantity is estimated by fitting the radioactivity prediction of Eq. (2) to the radioactivity measurements. The fitting is conducted by the least squares method, determining the coefficient to minimize the sum of squares of residual difference of predictions and measurements. When considering the uncertainty, the maximum-likelihood method is employed, determining the coefficient in such a way that the largest value of the likelihood function is acquired. Using these two methods, the estimation of intake is possible by solving the following equation:

$$
\min \|\boldsymbol{Q}(\boldsymbol{I})-\boldsymbol{M}\|_{2}^{2}
$$

where, \|\|$_{2}$ is the two-norm. For a measurement without uncertainty, $\boldsymbol{Q}(\boldsymbol{I})=\boldsymbol{Q} \cdot \boldsymbol{I}$ is determined and expressed with the matrix $\boldsymbol{Q}$ (component: $q\left(T_{i}-t_{j}\right)$ ), intake vector $\boldsymbol{I}$ (component: $\boldsymbol{I}\left(t_{j}\right)$ ), and measurement vector $\boldsymbol{M}$ (component: $\mathrm{m}\left(T_{j}\right)$ ). On the other hand, for a measurement with uncertainty, the component of $\boldsymbol{Q}(\boldsymbol{I})$ is $\left(\ln \left(\Sigma_{j} q\left(T_{i}-t_{j}\right) I\left(t_{j}\right)\right) / \ln \left(S F_{i}\right)\right.$, and the measurement vector $\boldsymbol{M}$ is (component: $\left.\ln \left(m\left(T_{i}\right)\right) / \ln \left(S F_{i}\right)\right) . S F_{i}$ represents a scattering factor ${ }^{3)}$ that indicates the uncertainty of the radioactivity measurement and corresponds to the geometric standard deviation in the logarithmic normal distribution.

For a measurement without uncertainty, Eq. (3) is a linear equation in terms of the intake vector $\boldsymbol{I}$. Therefore, the minimum intake vector $\boldsymbol{I}$ is acquired by solving the normal equation. For a measurement with uncertainty, Eq. (3) is not linear in terms of the intake vector $I$. Therefore, the intake vector $\boldsymbol{I}$ cannot be acquired as it is. Hence, Taylor expansion of $\boldsymbol{Q}(\boldsymbol{I})$ is conducted on the trial solution $\boldsymbol{I}^{k}$ and by approximating to the first order term $\left(\boldsymbol{Q}\left(\boldsymbol{I}^{k}+\Delta \boldsymbol{I}\right) \approx \boldsymbol{Q}\left(\boldsymbol{I}^{k}\right)+J\left(\boldsymbol{I}^{k}\right)\right.$, where $\Delta \boldsymbol{I}$ and $\boldsymbol{J}\left(\boldsymbol{I}^{k}\right)$ are the Jacobian matrixes to $\left.\boldsymbol{Q}(\boldsymbol{I})\right)$. Eq. (3) then becomes:

$$
\begin{aligned}
& \min \left\|\boldsymbol{J}\left(\boldsymbol{I}^{k}\right) \boldsymbol{I}^{k+1}-\hat{\boldsymbol{M}}\left(\boldsymbol{I}^{k}\right)\right\|_{2}^{2} \\
& \boldsymbol{I}^{k+1}=\boldsymbol{I}^{k}+\Delta \boldsymbol{I} \\
& \hat{\boldsymbol{M}}\left(\boldsymbol{I}^{k}\right)=\boldsymbol{M}-\boldsymbol{Q}\left(\boldsymbol{I}^{k}\right)+\boldsymbol{J}\left(\boldsymbol{I}^{k}\right) \boldsymbol{I}^{k}
\end{aligned}
$$

Eq. (4) is a linear equation with regard to $\boldsymbol{I}^{k+1}$, therefore, the minimum $\boldsymbol{I}^{k+1}$ is derived by solving the following normal equation: 


$$
\left(\boldsymbol{J}\left(\boldsymbol{I}^{k}\right)^{T} \boldsymbol{J}\left(\boldsymbol{I}^{k}\right)\right) \boldsymbol{I}^{k+1}=\boldsymbol{J}\left(\boldsymbol{I}^{k}\right)^{T} \hat{\boldsymbol{M}}\left(\boldsymbol{I}^{k}\right)
$$

Starting from a trial solution and by applying Eq. (5) repeatedly to successively update the intake vector, the most appropriate intake vector $\boldsymbol{I}$ is acquired. However, the solution is only available when the inverse matrix $\left(\boldsymbol{J}\left(\boldsymbol{I}^{k}\right)^{T} \boldsymbol{J}\left(\boldsymbol{I}^{k}\right)\right)^{-1}$ exists. An inverse matrix exists when the number of intakes (unknown number) is equal to or lower than the number of measurements (number of equations).

Further, a method is considered to solve Eq. (5) regardless of the dimensional relationship between the number of intakes and measurements.

\section{Inverse Analysis by Singular-Value Decomposition}

A singular-value decomposition of an arbitrary matrix $\mathrm{m} \times \mathrm{n}$ called matrix $\boldsymbol{A}$ is expressed as follows:

$$
\boldsymbol{A}=\boldsymbol{U} \boldsymbol{S} \boldsymbol{V}^{T}
$$

where matrix $\boldsymbol{U}$ is an $\mathrm{m} \times \mathrm{m}$ matrix consisting of column vectors of an orthonormal basis in the m-dimensional space, matrix $\boldsymbol{V}$ is an $\mathrm{n} \times \mathrm{n}$ matrix consisting of column vectors of an orthonormal basis in the n-dimensional space, and matrix $\boldsymbol{S}$ is an $\mathrm{m} \times \mathrm{n}$ diagonal matrix with singular values ( $s_{1} \geq \ldots \geq s_{i p} \geq 0, p$ : number of positive singular values) on its diagonal line. If the Jacobian matrix $\boldsymbol{J}$ of $\mathrm{m} \times \mathrm{n}$ in Eq. (5) is replaced by $\boldsymbol{J}=\boldsymbol{U} \boldsymbol{S} \boldsymbol{V}^{T}$ and coordinated using $\boldsymbol{U} \boldsymbol{U}^{T}=$ $\boldsymbol{U}^{T} \boldsymbol{U}=\boldsymbol{E}$ and $\boldsymbol{V} \boldsymbol{V}^{T}=\boldsymbol{V}^{T} \boldsymbol{V}=\boldsymbol{E}(\boldsymbol{E}$ : Unit matrix $)$, the following equation is obtained:

$$
\boldsymbol{I}^{k+1}=\boldsymbol{V}\left(\boldsymbol{S}^{T} \boldsymbol{S}\right)^{-1} \boldsymbol{S}^{T} \boldsymbol{U}^{T} \hat{\boldsymbol{M}}
$$

Considering matrix $\boldsymbol{S}^{T} \boldsymbol{S}$ being an $\mathrm{n} \times \mathrm{n}$ diagonal matrix, Eq. (7) is expressed as the following equation:

$$
\boldsymbol{I}^{k+1}=\sum_{i=1}^{p} \frac{\left(\boldsymbol{U}_{\cdot, i}\right)^{T} \hat{\boldsymbol{M}}}{s_{i}} \boldsymbol{V}_{\cdot, i}
$$

The calculation of the singular-value decomposition is possible with an arbitrary $\mathrm{m} \times \mathrm{n}$ matrix. Therefore, using Eq. (8), the estimation of the intake quantity is possible regardless of the dimensional relationship between the number of intakes $n$ and the number of measurements $\mathrm{m}$. Furthermore, the estimation of the intake quantity is possible using Eq. (8), not only for measurements with uncertainty, but also for measurements without uncertainty.

When the number of measurements $m$ is equal to or greater than the number of intakes $\mathrm{n}(\mathrm{m} \geq \mathrm{n})$, the result of the inverse analysis using the above singular-value decomposition is equal to the result using the least squares method. This is not the case for the opposite condition $(\mathrm{m}<\mathrm{n})$. The evaluation will be in the direction from the space of lower dimensions (m-dimensional space) to that of higher dimensions (n-dimensional space) that includes a certain bias on the inverse analysis. This bias can be determined with the difference between the intake quantity acquired by an inverse analysis considering the evaluated radioactivity prediction and through a direct analysis from specific intake quantities (e.g., 2.0 Bq, etc.) as the measured values and the original specific intake quantity. 


\section{Application Examples of Intake Estimations}

\section{Measurements without Uncertainty}

As an example of intake estimation applied to measurements without uncertainty, a case of inhalation of radioactive iodine by a worker described in a report from the International Atomic Energy Agency (IAEA) ${ }^{5)}$ was evaluated. In this report, thyroid radioactivity measurements of $480 \mathrm{kBq}$ and $440 \mathrm{kBq}$ obtained respectively on Wednesday and Thursday after inhalation of ${ }^{131} \mathrm{I}$ aerosol (AMAD $5 \mu \mathrm{m}$, Type-F) by a worker are presented.

The predicted thyroid radioactivity per unit intake required for estimating the intake quantity was determined as shown in Table 1. This example involves a case where the uncertainty of measurement is not considered, and the $\boldsymbol{Q}(\boldsymbol{I})$ in the Eq. (3) was expressed as a product of matrix $\boldsymbol{Q}$ listing the predicted thyroid radioactivity per unit intake and the intake quantity $\boldsymbol{I}$. Accordingly, the Jacobian matrix $\boldsymbol{J}$ became matrix $\boldsymbol{Q}$, and the intake quantity was calculated by the singular-value decomposition of matrix $\boldsymbol{Q}$ and Eq. (8). The estimation results are presented in Table 2. The first intake was estimated as the maximum of the three intakes. On the other hand, IAEA estimated the average of the three intakes, and the total intake quantity was determined to be $4,299 \mathrm{kBq}$. This is lower than our estimated total intake of $4.425 \mathrm{kBq}$ by approximately $2.8 \%$. The intake quantity on Friday, Monday, and Tuesday was calculated by inverse analysis using a predicted value acquired by direct analysis based on the assumption that if the intake quantity was $2.00 \mathrm{~Bq}$, each of the predicted values would be $1.70 \mathrm{~Bq}, 2.05 \mathrm{~Bq}$ and $2.17 \mathrm{~Bq}$ respectively. This result explains that a bias was added through inverse analysis. The predicted thyroid radioactivity calculated from the estimated result of the intake quantity is shown in Figure 1. The predicted values correspond to the measurements.

This example involved a problem of having to estimate the three intakes from two measurements and it could not be solved using the least squares method. However, by adopting this estimation method using Eq. (8), it was possible to solve this issue regardless of the dimensional relationship between the number of intakes and measurements.

Table 1 Predicted values per unit intake for inhalation of ${ }^{131} \mathrm{I}$ by worker

\begin{tabular}{cc}
\hline Time after intake $[\mathrm{d}]$ & AMAD $5 \mu \mathrm{m}($ Type $\mathrm{F})$ Thyroid $[\mathrm{Bq} / \mathrm{Bq}]$ \\
\hline 1 & $1.222 \times 10^{-1}$ \\
2 & $1.187 \times 10^{-1}$ \\
3 & $1.084 \times 10^{-1}$ \\
4 & $9.866 \times 10^{-2}$ \\
5 & $8.976 \times 10^{-2}$ \\
6 & $8.168 \times 10^{-2}$ \\
\hline
\end{tabular}

Table 2 Estimated intakes for inhalation of ${ }^{131} \mathrm{I}$ by worker

\begin{tabular}{cccc}
\hline \multirow{2}{*}{ Time after intake } & Estimated intakes $[\mathrm{Bq}]$ & \multicolumn{2}{c}{ Thyroid } \\
\hline Friday & $1.85 \times 10^{6}$ & & \\
Monday & $1.25 \times 10^{6}$ & & \\
Tuesday & $1.33 \times 10^{6}$ & & \\
Wednesday & & $4.80 \times 10^{5}$ & $4.76 \times 10^{5}$ \\
Thursday & & $4.40 \times 10^{5}$ & $4.44 \times 10^{5}$ \\
\hline
\end{tabular}




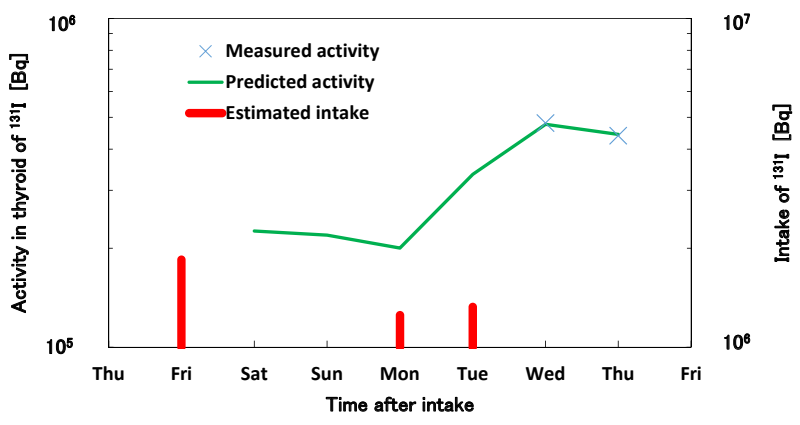

Figure 1 Predicted activity for inhalation of ${ }^{131} \mathrm{I}$ by worker

Table 3 Measured values of ${ }^{137} \mathrm{Cs}$ for worker

\begin{tabular}{cc}
\hline Time of measurement after intake $[\mathrm{d}]$ & Whole body $[\mathrm{Bq}]$ \\
\hline 1 & $8.8 \times 10^{4}$ \\
8 & $6.0 \times 10^{4}$ \\
15 & $3.0 \times 10^{4}$ \\
17 & $9.3 \times 10^{4}$ \\
30 & $8.3 \times 10^{4}$ \\
46 & $4.8 \times 10^{5}$ \\
50 & $4.6 \times 10^{5}$ \\
60 & $4.4 \times 10^{5}$ \\
\hline
\end{tabular}

\section{Measurements with Uncertainty}

A case of intake of ${ }^{137} \mathrm{Cs}$ by a worker shown in the IDEAS guidelines ${ }^{3)}$ was evaluated as an example of applying intake estimation to measurements with uncertainty. The worker (35-year-old male) was sprayed with liquid ${ }^{137} \mathrm{Cs}$ on his face when removing it from a bottle. The measured radioactivity on the entire body after exposure is presented in Table 3. The scattering factor was estimated as 1.2 for each measurement, and it was assumed that ingestion had occurred. The measurement taken on the 15th day was lower than the other measurements. It was therefore determined to be an outlier and not considered for evaluation. After the first intake, a second intake was assumed from an increase in the measurements taken between the 30th and 46th day, and the median day, the 38th, was considered as the day of intake.

The predicted radioactivity on the entire body per unit intake required for an intake estimation was evaluated, as presented in Table 4. As this example represents a measurement with uncertainly, and $\boldsymbol{Q}(\boldsymbol{I})$ from Eq. (3) was expressed using the predicted radioactivity on the entire body per unit intake, intake quantity, and logarithmic function of uncertainty. Accordingly, the intake quantity was calculated by performing singular-value decomposition from the acquired Jacobian matrix $\boldsymbol{J}$ and by the repeated application of Eq. (8). The estimated results are presented in Table 5. On the other hand, according to the IDEAS guidelines estimation, values of $93,988 \mathrm{~Bq}$ on the 0 th day and $479,100 \mathrm{~Bq}$ on the 38 th day were determined, corresponding to smaller values than ours by approximately $1 \%$. Furthermore, the intake quantity calculated with the predicted values using direct analysis based on an assumption of $2.00 \mathrm{~Bq}$ for the first and second intake quantities was $2.00 \mathrm{~Bq}$ and $2.00 \mathrm{~Bq}$, respectively, demonstrating the absence of bias in an inverse analysis. The predicted radioactivity on the entire body evaluated from the estimation result is presented in Figure 2. The predicted values match the trend of measurements. 
Table 4 Predicted values per unit intake for ingestion of ${ }^{137} \mathrm{Cs}$ by worker

\begin{tabular}{cc}
\hline Time after intake $[\mathrm{d}]$ & Whole body $[\mathrm{Bq} / \mathrm{Bq}]$ \\
\hline 1 & $9.801 \times 10^{-1}$ \\
8 & $8.596 \times 10^{-1}$ \\
12 & $8.317 \times 10^{-1}$ \\
15 & $8.146 \times 10^{-1}$ \\
17 & $8.040 \times 10^{-1}$ \\
22 & $7.785 \times 10^{-1}$ \\
30 & $7.398 \times 10^{-1}$ \\
46 & $6.682 \times 10^{-1}$ \\
50 & $6.514 \times 10^{-1}$ \\
60 & $6.112 \times 10^{-1}$ \\
\hline
\end{tabular}

Table 5 Estimated intakes for ingestion of ${ }^{137} \mathrm{Cs}$ by worker

\begin{tabular}{cccc}
\hline Time after intake [d] & Estimated intakes [Bq] & \multicolumn{2}{c}{ Whole body } \\
\hline 0 & $9.50 \times 10^{4}$ & & \\
1 & & $8.8 \times 10^{4}$ & $9.31 \times 10^{4}$ \\
8 & & $6.0 \times 10^{4}$ & $8.16 \times 10^{4}$ \\
15 & $3.0 \times 10^{4 a)}$ & $7.74 \times 10^{4}$ \\
17 & & $9.3 \times 10^{4}$ & $7.64 \times 10^{4}$ \\
30 & & $8.3 \times 10^{4}$ & $7.03 \times 10^{4}$ \\
38 & & \\
46 & $4.85 \times 10^{5}$ & $4.8 \times 10^{5}$ & \\
50 & & $4.6 \times 10^{5}$ & $4.80 \times 10^{5}$ \\
60 & & $4.4 \times 10^{5}$ & $4.65 \times 10^{5}$ \\
\hline
\end{tabular}

a) outlier

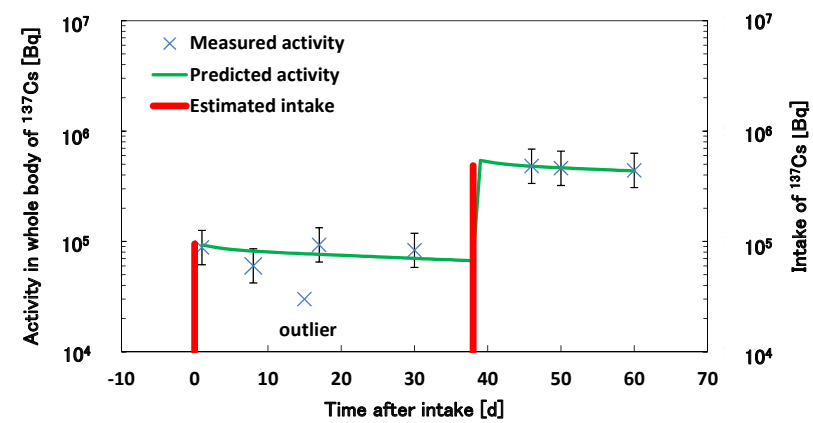

Figure 2 Predicted activity for ingestion of ${ }^{137} \mathrm{Cs}$ by worker

In this case, multiple intakes were evaluated considering the uncertainty of the measurements represented by the scattering factor. Using this estimation method, the evaluation of multiple intakes was possible for measurements with uncertainty, similarly to single intakes. 


\section{Conclusions}

To conduct a highly reliable dose evaluation of internal exposure, it was required to adequately estimate the level of intake. When an unexpected intake of radioactive materials occurs, the intake quantity is unknown and is therefore estimated by inverse calculation from the internal radioactivity or excretions, measured after intake. Estimation of the intake quantity was conducted by fitting the predicted radioactivity to the measured radioactivity values and was formulated as a least squares problem. To employ the least squares method, the number of intakes must be lower than the number of measurements. From the ICRP ${ }^{2)}$ and IDEAS ${ }^{3)}$ guidelines, only a single intake was considered, and the estimation of multiple intakes was not sufficiently conducted. Using the intake estimation method with singular-value decomposition in this research, estimation was possible regardless of the dimensional relationship between the number of intakes and the number of measurements. The effectiveness of this estimation method was demonstrated by applying it to case examples of intakes from previous studies.

Generally, for a measurement with uncertainty, the uncertainty is propagated to the results with inverse analyses. Accordingly, when the reliability of the analysis results are considered, it is essential to evaluate the propagation of uncertainty. Herein, a method permitting the evaluation regardless of dimensional differences between the number of measurements and the number of intakes was proposed. It will be necessary to consider the propagation of uncertainty to target evaluations with a higher degree of accuracy in the future.

\section{References}

1) ICRP, “1990 Recommendations of the international commission on radiological protection," ICRP Publication 60, Ann. ICRP 21 (1-3) (1991).

2) ICRP, "Individual monitoring for internal exposure of workers," ICRP Publication 78, Ann. ICRP 27 (3-4) (1997).

3) C. M. Castellani, J. W. Marsh, C. Hurtgen, E. Blanchardon, P. Berard, A. Giussani, M. A. Lopez, "IDEAS guidelines (version 2) for the estimation of committed doses from incorporation monitoring data," EURADOS Rep. 2013-01 (2013).

4) R. C. Aster, B. Borchers, C. H. Thurber, "Parameter estimation and inverse problems," Elsevier Academic Press ISBN: 0123850487 (2012).

5) IAEA, "Methods for assessing occupational radiation doses due to intakes of radionuclides," Safety Rep. Series No. 37 (2004). 